\title{
Clinical diagnosis by junior doctors - How confident and accurate are they?
}

\author{
Sudusinghe $D H^{1}$, Indrakumar $\mathcal{J}^{2}$
}

\begin{abstract}
Background: Accurate diagnosis is necessary for effective treatment. Over the past few years it has been noticed that the junior doctors are not confident enough to make a diagnosis after initial history and examination. Aim of this study is to determine the ability of junior doctors to document a clinical diagnosis and accuracy of the diagnosis.

Method: This single centre study included case records of patients admitted to Professorial Medical Unit (PMU) and Emergency Treatment Unit (ETU) at Colombo South Teaching Hospital (CSTH). The junior doctors' on admission diagnosis is compared with the medical consultants' diagnosis. Only case records of patients belonging to four common specialty domains were studied.

Results: In the PMU out of 200 case records no diagnosis or symptom as a diagnosis was documented in $99(49.5 \%)$ cases and a diagnosis was documented in 68(34.0\%) case records of which $53(77.9 \%)$ diagnoses were concordant with the medical consultants' diagnosis. When case records of patients admitted to ETU considered, no diagnosis or symptom as a diagnosis was documented in $56(56.0 \%)$ case records and a diagnosis was documented in only $21(21.0 \%)$ case records of which $15(71.4 \%)$ diagnoses were concordant with the medical consultants' diagnosis. Documentation of correct diagnosis improved with the grade of the doctor, from intern medical officer (IMO) to medical registrar (MR) in both study settings and also with the order of clerking.
\end{abstract}

Conclusion: Recording of symptom based diagnosis or no diagnosis remains high among most of the junior doctors in all specialty domains and at all grades.

Key words: Junior doctor, diagnosis, common medical condition

\section{Background}

The difference between a doctor and other health care personnel is the ability of the doctor to work as a clinical scientist who is able to apply the principles and procedures of medicine to diagnose and treat patients (The Medical Schools Council, 2008). Ability of diagnosis varies among individual doctors, and greatly depends on the knowledge and experience of the doctor.

${ }^{1}$ Department of Physiology, Faculty of Medical Sciences, University of Sri Jayewardenepura, Sri Lanka

${ }^{2}$ Department of Medicine, Faculty of Medical Sciences, University of Sri Jayewardenepura, Sri Lanka

Corresponding author:

Dr. Dinesha Himali Sudusinghe

Department of Physiology, Faculty of Medical Sciences, University of Sri Jayewardenepura, Sri Lanka

Email:dinesha94@yahoo.com
While easy cases can be diagnosed by identifying the patterns of presentation, difficult diagnostic problems can be solved by generating a limited number of hypotheses early in the diagnostic process. Those hypotheses can be used to guide subsequent investigations (Elstein, 2002). Comprehensive clinical history and examination is essential to identify patterns of presentation and to formulate reasonable hypotheses. The doctor should be able to make a reasonable diagnosis by critically analysing the information that they gather from the initial history and examination.

The diagnostic ability and the accuracy of the diagnosis can be influenced by several factors. The ability of doctor to communicate effectively with the patient is one of the important factors (Leavitt \& Leavitt, 1970).Published guidance has stressed the 
importance of the doctors to show their performance and competence when treating patients and also the importance of identifying non-verbal communication during the medical consultation (General Medical Council, 2009).

There have been many advances in the diagnostic techniques for most diseases. However, taking a detailed and accurate history and performing a proper clinical examination still remains important as it is adequate to lead to a diagnosis in more than $75 \%$ of patients (Peterson et al., 1992; Hampton et al., 1975). It has been observed that many junior doctors are not documenting a diagnosis or a differential diagnosis following clerking of acutely ill patients. A single centre audit done in UK has shown that the ability to make a diagnosis or a differential diagnosis remains suboptimal regardless of the specialty even among relatively experienced junior doctors (Bhandari, 2009). Current work place conditions and the training structures may have contributed to this.

Studies regarding diagnostic ability of junior doctors in Sri Lanka have not been published before. The aim of this study is to see whether the junior doctors are able to identify the correct system involved, ability to complete their clinical assessment by documenting a reasonable diagnosis, accuracy of their diagnosis when compared with the final diagnosis and the diagnostic ability in relation to grade of the doctor and order of clerking.

\section{Methods}

This retrospective single centre study was carried out in a Teaching Hospital in Sri Lanka. We analysed case records of patients admitted to the Professorial Medical Unit (PMU) and the Emergency Treatment Unit (ETU) at the Colombo South Teaching Hospital (CSTH) for two consecutive months. In both these settings patients were seen by different groups of doctors, including intern medical officer (IMO), medical officer emergency treatment unit (MO-ETU), senior house officer (SHO), medical registrar (MR) and medical consultant. All groups of doctors except consultants were identified as junior doctors. Junior doctors were graded according to seniority and post graduate qualifications. Thus, MO-ETU/SHO was at a higher grade than IMO due to seniority and MR was graded higher than MO-ETU and SHO due to postgraduate qualifications. Data were obtained from the case records of patients available at the record room at CSTH. The details of clinical diagnosis documented by junior doctors who clerked the patient initially were obtained together with the final documented diagnosis. Our study methodology is similar to the single centre audit conducted in UK to assess the diagnostic activity of junior doctors (Bhandari, 2009).

The four commonest specialty domains of clinical cases were identified based on hospital statistics (Cardiovascular, Gastroenterology /hepatology, Respiratory and Neurology). The case records under the above specialty domains were identified according to the symptomatology related to the relevant system. For example, the patients presenting with exertional chest pain and autonomic symptoms were grouped in the cardiovascular domain and patients with pleuritic chest pain and productive cough were grouped in the respiratory domain. Case records of all elective admissions, deaths and those who did not have symptoms related to selected specialty domains were excluded from the study. For convenience, only case records of patients with a hospital stay of less than five days were selected.

According to CSTH admission policy, patients admitted to PMU will be first seen by IMO, $\mathrm{SHO}$ or $\mathrm{MR}$ in the ward and all patients admitted to ETU will be first seen by the MOETU. If further opinion is needed, MO-ETU will refer the patient to the casualty medical ward MR or if further opinion is not needed the patient is directly transferred to the casualty medical ward after initial management and once the patient is stable. Thus, IMO and MR will be seeing the ETU patients after MO-ETU as a second clerking.

Data collection was carried out until 50 case records for each specialty domain in the PMU and 25 case records for each specialty domain in the ETU were completed. Descriptive statistical methods (tables and charts) were used to describe data. Cross tabulations was used to find out the association between the categorical variables and Chi squared test was used to assess the significance. $P$ value $<0.05$ was considered significant. All analysis was done using SPSS statistical package 11.

\section{Results}

Case records of two hundred patients admitted to PMU and one hundred patients admitted to ETU were analyzed. There were 181 males and 119 females aged 15-99 years with a mean of 68.5 years. 


\section{Confidence and accuracy of junior doctors' diagnosis in PMU}

Of 200 case records of patients at the PMU, correct specialty domain was identified in $167(83.5 \%)$. No diagnosis or a symptom as a diagnosis (e.g. haematemesis, chest pain) was given for $99(49.5 \%)$ case records. A diagnosis was documented in only $68(34.0 \%)$ case records of which 53(77.9\%) were accurate and concordant with the medical consultants' diagnosis. Out of total number of cases analyzed, the junior doctors were able to document a correct diagnosis only in $26.5 \%$ of case records (Table 1). Documentation of correct system and correct diagnosis according to the different grades of the doctor (IMO and MR) are summarized in Figure 1. Details of documentation by the SHO were excluded since there were only 7 case records of patients which carried their diagnosis.

No significant association was found between ability of identification of the correct system and grade of the doctor $(P>0.05)$. When junior doctors' ability to identify the correct system was considered (Table 2), significant association was found, with the proportion of the cases correctly documented by the MR being significantly higher than that of the IMO $(\mathrm{P}<0.001)$.

Table 1: Professorial medical unit diagnostic conclusions

\begin{tabular}{|c|c|c|c|c|c|c|c|c|c|c|}
\hline \multirow[t]{2}{*}{$\begin{array}{l}\text { Specialty } \\
\text { domain }\end{array}$} & \multirow{2}{*}{ 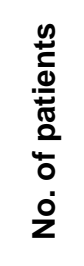 } & \multicolumn{2}{|c|}{$\begin{array}{l}\text { Correct } \\
\text { system }\end{array}$} & \multicolumn{2}{|c|}{$\begin{array}{l}\text { No diagnosis } \\
\text { or symptom } \\
\text { only diagnosis }\end{array}$} & \multicolumn{2}{|c|}{$\begin{array}{c}\text { Diagnosis } \\
\text { documented }\end{array}$} & \multicolumn{2}{|c|}{$\begin{array}{c}\text { Correct } \\
\text { diagnosis }\end{array}$} & \multirow{2}{*}{ 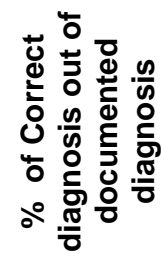 } \\
\hline & & $\mathrm{n}$ & $\%$ & $\mathrm{n}$ & $\%$ & $\mathrm{n}$ & $\%$ & $\mathrm{n}$ & $\%$ & \\
\hline Cardiovascular & 50 & 42 & $84 \%$ & 24 & $48 \%$ & 18 & $36 \%$ & 14 & $28 \%$ & $77.8 \%$ \\
\hline Respiratory & 50 & 44 & $88 \%$ & 24 & $48 \%$ & 20 & $40 \%$ & 16 & $32 \%$ & $80.0 \%$ \\
\hline Gastroenterology & 50 & 38 & $76 \%$ & 27 & $54 \%$ & 11 & $22 \%$ & 6 & $12 \%$ & $54.5 \%$ \\
\hline Neurology & 50 & 43 & $86 \%$ & 24 & $48 \%$ & 19 & $38 \%$ & 17 & $34 \%$ & $89.5 \%$ \\
\hline Total & 200 & 167 & $83.5 \%$ & 99 & $49.5 \%$ & 68 & $34 \%$ & 53 & $26.5 \%$ & $77.9 \%$ \\
\hline
\end{tabular}

Figure 1: Percentage of doctors of different grades and their ability to identify correct system and making a correct diagnosis in the Professorial Medical Unit

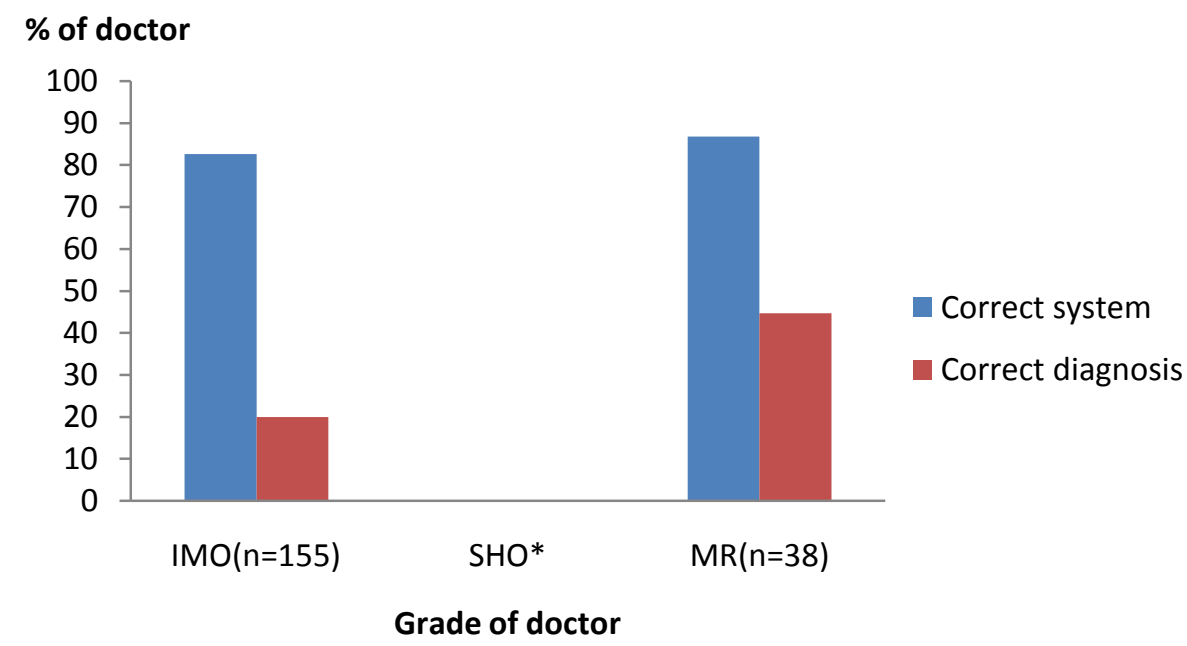

${ }^{*}$ For SHO there were only seven cases seen and therefore the data were not interpretable 
Table 2: Relationship between ability of documentation diagnosis and grade of doctor in PMU

\begin{tabular}{|c|c|c|c|c|c|c|}
\hline \multirow{3}{*}{ Documented diagnosis } & \multicolumn{4}{|c|}{ Grade } & \multirow{2}{*}{\multicolumn{2}{|c|}{ Total }} \\
\hline & \multicolumn{2}{|c|}{ IMO } & \multicolumn{2}{|c|}{ MR } & & \\
\hline & $\mathbf{n}$ & (\%) & $\mathbf{n}$ & (\%) & $\mathbf{n}$ & $(\%)$ \\
\hline Correct & 31 & 24.2 & 17 & 51.5 & 48 & 29.8 \\
\hline Wrong & 8 & 6.3 & 7 & 21.2 & 15 & 9.3 \\
\hline $\begin{array}{l}\text { No diagnosis detailed or } \\
\text { symptom only }\end{array}$ & 89 & 69.5 & 9 & 27.3 & 98 & 60.9 \\
\hline Total & 128 & 100.0 & 33 & 100.0 & 161 & 100.0 \\
\hline
\end{tabular}

$\mathrm{X}^{2} 20.558, \mathrm{df}=2, \mathrm{P}<0.001$

Note: Numbers do not add up to 200 since wrong system was identified in thirty three cases and six cases were seen by the $\mathrm{SHO}$ and are not included for the above analysis.

\section{Confidence and accuracy of junior doctors' diagnosis in ETU}

When first clerking doctors(MO-ETU) were considered, out of one hundred case records of patients, correct specialty domain was given for $87(87.0 \%)$ and no diagnosis or symptom as a diagnosis was given for $56(56.0 \%)$. A diagnosis was documented in $21(21.0 \%)$ case records of which $15(71.4 \%)$ were accurate and concordant with the medical consultants' diagnosis. Out of total number of cases analyzed, MO-ETU was able to document a correct diagnosis only in $15.0 \%$ of case records. With regard to second clerking
(IMO/MR), correct specialty domain was given for $94(94.0 \%)$ case records and no diagnosis or symptom as a diagnosis was given for $46(46.0 \%)$. A diagnosis was documented in only $48(48.0 \%)$ case records of which $41(85.4 \%)$ diagnoses were accurate and concordant with the medical consultants' diagnosis. Second clerking doctors were able to document a correct diagnosis in $41 \%$ of case records out of total number of cases analyzed. Documentation of correct system and correct diagnosis according to the different grades of the doctor (IMO, MO-ETU and MR) is summarized in (Figure 2).

Figure 2: Percentage of doctors of different grades and their ability to identify correct system and making a correct diagnosis in Emergency Treatment Unit

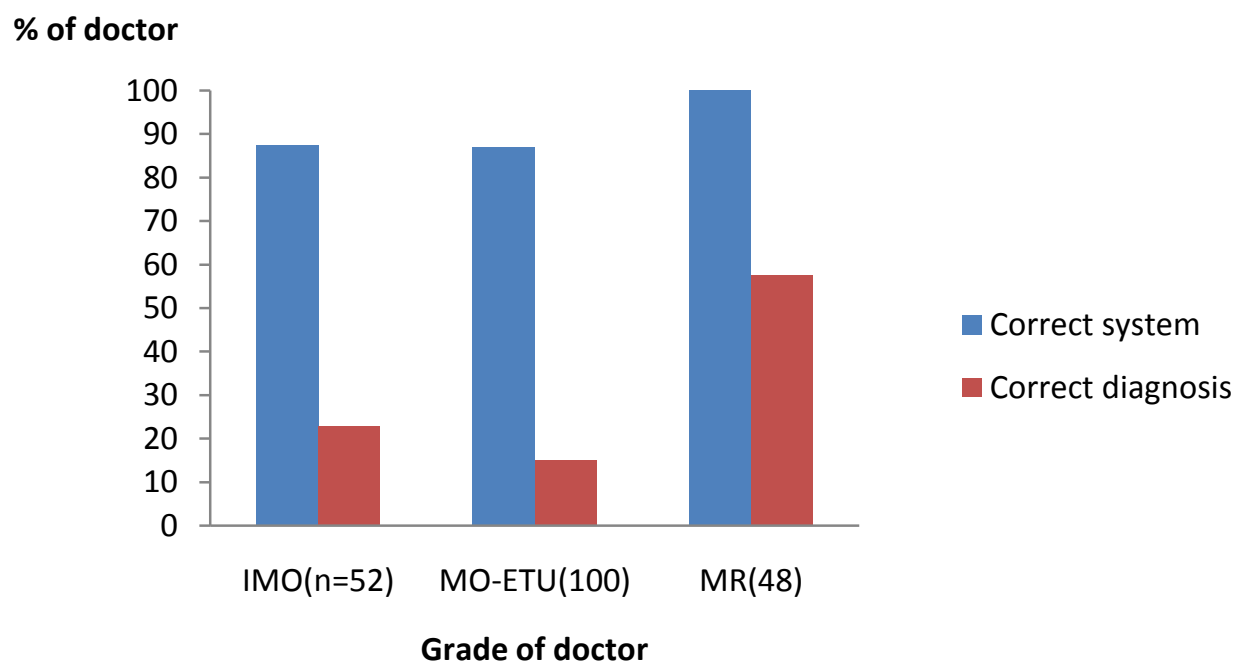

* All the cases seen by the Mo-ETU were seen by IMO and MR as a second clerking doctor 
There was a significant association between the grade of the second clerking doctor and their ability to identify the correct system $(P<0.05)$. However, when junior doctors ability of documentation of diagnosis and the grade of the junior doctor considered there was a significant association $(P<0.05)$ where, the proportion of the cases correctly diagnosed and documented by the MR was higher than that of the IMO (Table 3). Ability of documentation of correct system and correct diagnosis according to the order of clerking is summarized in (Figure 3).

Table 3: Relationship between ability of documentation of correct diagnosis and grade of second clerking doctor in ETU

\begin{tabular}{cccccccc}
\hline & \multicolumn{3}{c}{ Grade } & \multicolumn{2}{c}{ Total } \\
Documented diagnosis & $\mathbf{n}$ & IMO & $\mathbf{n}$ & (\%) & $\mathbf{n}$ & $(\%)$ \\
\hline Correct & 11 & 26.2 & 30 & 57.7 & 41 & 43.6 \\
Wrong & 4 & 9.5 & 3 & 5.8 & 7 & 7.4 \\
No diagnosis detailed or & 27 & 64.3 & 19 & 36.5 & 46 & 48.9 \\
symptom only & $\mathbf{4 2}$ & $\mathbf{1 0 0}$ & $\mathbf{5 2}$ & $\mathbf{1 0 0}$ & $\mathbf{9 4}$ & $\mathbf{1 0 0}$ \\
Total & & & & & &
\end{tabular}

$x^{2} 9.381, d f=2, P<0.05$

Note: Numbers do not add up to 100 since wrong system was identified in six cases and are not included for the above analysis

Figure 3: Percentage of identifying correct specialty system and making a correct diagnosis according to order of clerking in Emergency Treatment Unit

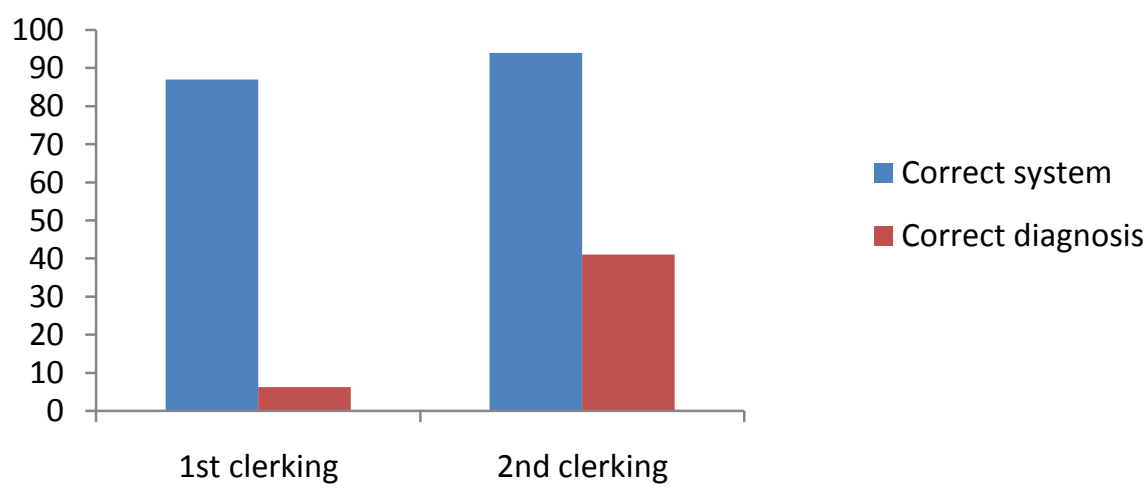

\section{Discussion}

In this study 200 case records from PMU and 100 case records from ETU were analyzed. In both PMU and ETU almost all junior doctors have shown the ability to identify the correct specialty domain in majority of cases (PMU $83.5 \%$, ETU $87.0 \%)$.In the PMU although most $(83.5 \%)$ junior doctors identify the correct specialty domain, almost half $(49.5 \%)$ of the case records were given no diagnosis or symptom as a diagnosis. Only $26.5 \%$ of diagnoses were concordant with the medical consultants' diagnosis. It suggests that although most of the junior doctors were able to identify the specialty domain correctly they were not confident enough to document a reasonable diagnosis or differential diagnosis after the clinical history and examination. The doctor is a clinical scientist who applies the principles and procedures of medicine to diagnose and to treat the patients with disease 
and injuries to maintain their physical and mental health (The Medical Schools Council, 2008). To achieve this doctor should be able to apply their knowledge and skills to make a diagnosis or differential diagnosis following clerking of new patients. Lower percentage of documentation of diagnosis in this study may be due to lack of confidence to write the clinical diagnosis or due to possible lack of sufficient knowledge regarding the illness. A similar study done in UK has observed how junior doctors complete and document fundamental diagnostic assessments in an acute care setting. The results were similar to our study where most of the junior doctors did not document a differential diagnosis based on their history and examination despite programmed training and irrespective of its defined accuracy. According to our results UK doctors were able to diagnose better (45\%) than our junior doctors (26.5\%) in the acute medical assessment unit (Bhandari, 2009).

The PMU doctors identified a correct diagnosis for $53(77.9 \%)$ case records out of the 68 cases when they had attempted to document a diagnosis. It suggests that if the doctor attempted to document a reasonable diagnosis, there is a possibility that most of the time junior doctors' diagnosis can be concordant with the admitting medical consultants' diagnosis. Uncertainties within the minds of the individual junior doctor fearing of an incorrect diagnosis and being criticized by their senior doctors could be one reason for the lack of documentation of a diagnosis.

In the PMU, there was no significant difference in identification of correct system with increasing grade. However, there was a significant improvement in the documentation of correct diagnosis with the increase grade of the junior doctor. It appears that less experienced doctors are more comfortable with recording a symptom as a diagnosis rather than a diagnosis when it comes to documenting their diagnostic conclusion. However, recording of symptom based diagnosis remains high among all junior doctors irrespective of specialty domains and grade of the doctor.

The patients admitted to ETU are likely to be more ill with a specific complaint than PMU patients who are more likely to have less acute and more nonspecific symptoms. Therefore identification of specialty domain and the diagnosis in the ETU patients is likely to be easier than with patients admitted to the PMU.The diagnosis was correctly documented in $15 \%$ of case records of patients admitted to ETU, whereas a correct diagnosis was given for $26.5 \%$ of case records of patients admitted to PMU. The busier working environment in the ETU may have contributed to the lower percentage of documentation of diagnosis observed above.

In the ETU, second clerking doctors were able to identify the correct system and document a correct diagnosis more accurately than the first clerking doctors. The stepwise information gained by the second clerking doctors may have contributed to this difference observed.

Sri Lanka, being a developing country, the facilities available in the government hospitals remains inadequate. Most of the drugs in the essential drug list are available in government hospitals free of charge but uniform availability throughout the year is not guaranteed (World Health Organization, 2007).To prevent the inappropriate use of available facilities, it is important to make a specific and early diagnosis. Therefore the doctor should be able to make a reasonable differential diagnosis by the history and examination. Inappropriate use of drugs as well as other facilities maybe minimized by improving the junior doctors' ability and confidence of making a proper clinical diagnosis.

Junior doctors' initial diagnosis was compared with the final diagnosis which is most commonly the medical consultants' diagnosis. Although the consultant's diagnosis is considered most accurate, post mortem studies have shown diagnostic errors could occur in the range of $10-20 \%$ of patients (Shojania, 2003).

This study is mainly aimed at finding the confidence and accuracy of documentation of a correct diagnosis among casualty admissions. If the junior doctor clearly documents the most likely diagnosis or differential diagnosis after the clinical assessment, the consultant can use this information to establish the correct diagnosis and start necessary treatment without delay. Diagnostic standards can be improved by observing and questioning others who make diagnoses and also establishing more continuity of care of patients for junior doctors and allowing them to follow patients through to final discharge (Bhandari, 2009).

Variable educational standards and teaching structures of different universities can influence the doctor's performance. Group of 
doctors in this study graduated from different universities. We have not attempted find out the effect of variable educational standards of different universities on their diagnostic activity. Even though all medical schools in UK have to incorporate the recommendations of Tomorrow's Doctors in to their curricula, outcomes of different medical schools in relation to postgraduate exam success have shown remarkable differences (McManus et al., 2008; Bowhay et al., 2009).

Gaining experience will take time but feedback by the consultants can help the junior doctor to improve their diagnostic abilities and standard (Levy et al., 2007). Even though the post casualty ward round is busy, giving more chances during the ward round for case presentations will help them immensely to gain experience. Today the role of the doctor is changing and will continue to change alongside the needs and expectations of patients. However, the fundamental clinical role of a junior doctor remains unchanged where the doctor is expected to take a good history, perform a complete examination and document a reasonable differential diagnosis.

\section{Limitations}

This is a single centre study of limited number of patients and a small sample of junior doctors. In order to avoid bias, the study should include a broad range of cases and varied range of medical officers. Case records included in this study belong to four common specialty domains and the doctors' diagnostic ability was not assessed on rare clinical conditions. The junior doctors in this study were graduates of different medical schools in Sri Lanka which may have affected the diagnostic ability of the individual doctor.

\section{Conclusions}

In both PMU and ETU most of the junior doctors were able to identify the correct specialty domain. Recording of symptom based diagnosis remains high among most of the junior doctors in all specialty domains and at all grades. However, significant improvement was observed with the increasing grade of the doctor, from IMO to MR regarding documentation of the correct diagnosis irrespective of the specialty domain in both settings.

\section{Acknowledgement}

We are very grateful to Dr.Wijayasiri, MBBS, MSC in Epidemiology, Senior Lecturer, Department of Community Medicine, Faculty of Medical Sciences, University of Sri Jayewardenepura for help with statistics.

\section{References}

Bhandari, S. (2009) A single-center audit of junior doctors' diagnostic activity in medical admissions, Journal of Royal College Physicians of Edinburgh, 39, pp. 307-312.

Bowhay, A.R. \& Watmough, S.D. (2009) An evaluation of the performance in the UK Royal College of Anaesthetists primary examination by UK medical school and gender, BMC Med Education, 9, 38, Available from: http://www. biomedcentral.com/1472-6920/9/38_doi:10.1186 11472-6920-9-38[Accessed on 19 July 2014].

Elstein, A.S. \& Schwarz, A. (2002) Clinical problem solving and diagnostic decision making: selective review of the cognitive literature, British Medical Journal, 324, pp. 729-732.

General Medical Council. (2009) Tomorrow's doctors: recommendations on undergraduate medical education, London: General Medical Council.

Hampton, J.R., Harrison, M.J., Mitchell, J.R, Prichard, J.S. \& Seymour, C. (1975) Relative contributions of history-taking, physical examination, and laboratory investigation to diagnosis and management of medical outpatients, British Medical Journal, 2, 5969, pp. 486-489.

Leavitt, J. \& Leavitt, F. (2011) Improving medical outcomes: The psychology of doctor-patient visit, United Kingdom: Rowman \& Littlefield Publishers.

Levy, P.D. \& Sherwin, R. L., Kuhn, G.J. (2007) Clinical diagnostic reasoning, New England Journal of Medicine 356, pp.12721274.doi:10.1056/ NEJMc063606.

McManus, I.C., Elder, A.T., Champlain, A.D., Dacre, J.E., Mollon, J. \& Chis, L. (2008) Graduates of different medical schools show substantial differences in performance in MRCP Part1, Part 2 and PACES examinations, BMC Medicine, 6:5. Available from: http://www.biomedcentral.com /1741-7015/6/5doi:10.1186/1741-7015-6-5 [Accessed $19^{\text {th }}$ July 2014].

Peterson, M.C., Holbrook, J.H., Von Hales, D., Lee Smith, N. \& Staker, L.V.( 1992) Contributions of the history, physical examination and laboratory investigation in making medical diagnoses, Western Journal of Medicine, 56, pp. 163-165. 
Shojania, K.G., Burton, E.C., McDonald, K.M. \& Goldman, L. (2003) Changes in rates of autopsydetected diagnostic errors over time: a systematic review, Journal of the American Medical Association 289, pp. 2849-2856.

The Chief Medical Officers of England, The Medical Schools Council (2008) The consensus statement on the role of the doctor, Available from: http://www.medschools.ac.uk/AboutUs/Projects/D ocuments/Role\%20of\%20Doctor\%20Consensus $\% 20$ Statement.pdf [Accessed $16^{\text {th }}$ Oct 2011].
World Health Organization (2007) 11 Health questions about 11 SEAR Countries, India: WHO Regional Office for South-East Asia, Available from: http://www.searo.who.int/en/section1243. htm [Accessed on 16 Oct 2011]. 